\title{
Dynamic properties of stainless steel under direct tension loading using a simple gas gun
}

\author{
Anatoly Bragov ${ }^{1, *}$, Alexander Konstantinov ${ }^{1}$, Leopold Kruszka $^{2}$, Andrey Lomunov ${ }^{1}$, and Andrey Filippov ${ }^{1}$ \\ ${ }^{1}$ Research Institute of Mechanics, National Research Lobachevsky State University of Nizhny Novgorod, 603022, Russia \\ ${ }^{2}$ Military University of Technology, Civil Engineering and Geodesy Faculty, 00-908 Warsaw, Poland
}

\begin{abstract}
The combined experimental and theoretical approach was applied to the study of high-speed deformation and fracture of the 1810 stainless steel. The material tests were performed using a split Hopkinson pressure bar to determine dynamic stress-strain curves, strain rate histories, plastic properties and fracture in the strain rate range of $10^{2} \div 10^{4} \mathrm{~s}^{-1}$. A scheme has been realized for obtaining a direct tensile load in the SHPB, using a tubular striker and a gas gun of a simple design. The parameters of the JohnsonCook material model were identified using the experimental results obtained. Using a series of verification experiments under various types of stress-strain state, the degree of reliability of the identified mathematical model of the behavior of the material studied was determined.
\end{abstract}

\section{Introduction}

It is known that the mechanical characteristics of materials can depend significantly on the rate of deformation. To determine the properties of materials at high strain rates $\left(10^{2} \div 10^{4} \mathrm{~s}^{-1}\right)$, the Kolsky method or the split Hopkinson pressure bar (SHPB) are widely used. The range of strain rates in which this method is applicable is of practical interest, since it is in such a high-speed regime that the structural elements are deformed in conditions of pulsed loading under impacts. The classical SHPB version for compression tests is the most researched, theoretically justified and most common, however, this kind of tests does not allow determining a number of such practically important strength and deformation characteristics of materials, such as temporary fracture resistance and ultimate plasticity characteristics. In addition, the behavior of the material can differ significantly for different types of stress-strain state, therefore the definition of stress-strain curves under tension is also an important independent task.

Various schemes of realized installations for direct dynamic tension of samples using the technique of pressure bars can be found in [1-5]. The main differences between these modifications rely on the method of generating a tensile impulse load. Various modifications of the indirect method of creating a tensile load are also known [6]. The sample is then connected to the measuring bars by means of a threaded or glued joint. The advantage of the direct method is a more qualitative picture of the detected strain pulses and, accordingly, greater reliability and accuracy of the obtained characteristics.

\section{Experimental methods and specimens}

A traditional version of the Kolsky method using SHPB and a gas gun was used to study the properties of steel under compression. To obtain a direct tensile load in the SHPB, the gas gun of a simple design was used (Fig. 1).

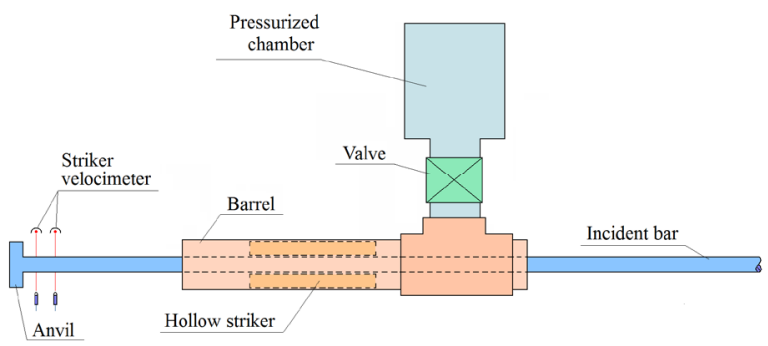

Fig. 1. Scheme of a gas gun for creating a direct tensile wave.

The sets of measuring bars with a diameter of $20 \mathrm{~mm}$ for compression and tension tests were made of highstrength maraging steel. Strain measurement was made by using low-base foil strain gauges glued on the side surface of measuring bars. To compensate for the flexural vibrations in the bars and to increase the amplitude of the useful signal, 4 connected in series strain gauges were glued in the working sections. Repeated tests were carried out under each loading regime (strain rate, temperature). Based on the series of charts obtained, for each test regime, an average curve with scatter characteristics of the experimental data (confidence intervals) was determined. The averaging of the dependences $\sigma \sim t, \varepsilon \sim t$ and $\dot{\boldsymbol{\varepsilon}} \sim t$ was carried out on a common time axis. Statistical processing was performed and confidence intervals were determined. 
The change in the strain rate of the sample was ensured by varying the striker velocity, and the required degree of deformation of the sample was achieved by varying the length of the striker. To study the behaviour of the material at elevated temperatures, a miniature furnace of a tubular structure was used, which was put on the ends of the measuring bars with the specimen placed between them. A miniature thermocouple welded to the side surface of the sample was used to control the temperature of the sample. At test temperatures up to $+350^{\circ} \mathrm{C}$, no correction of the formulas was carried out, since at these temperatures there had been no noticeable changes in the elastic characteristics of the bar material (elastic wave velocity and modulus of elasticity).

To conduct experimental studies, samples were made of the 1810 stainless steel in the form of cylinders with a diameter of $14 \mathrm{~mm}$ and a height of $7 \mathrm{~mm}$ (for compression testing) and in the form of entire samples with threaded heads with a working part by $10 \mathrm{~mm}$ in length and $5 \mathrm{~mm}$ in diameter (for tensile tests) .

\section{Experimental results and discussion}

A series of static and dynamic basic tests of steel in a wide range of strain rates and temperatures during compression and tension was carried out. The resulting average diagrams for compression are shown in Fig. 2, and for tension - in Fig. 3.

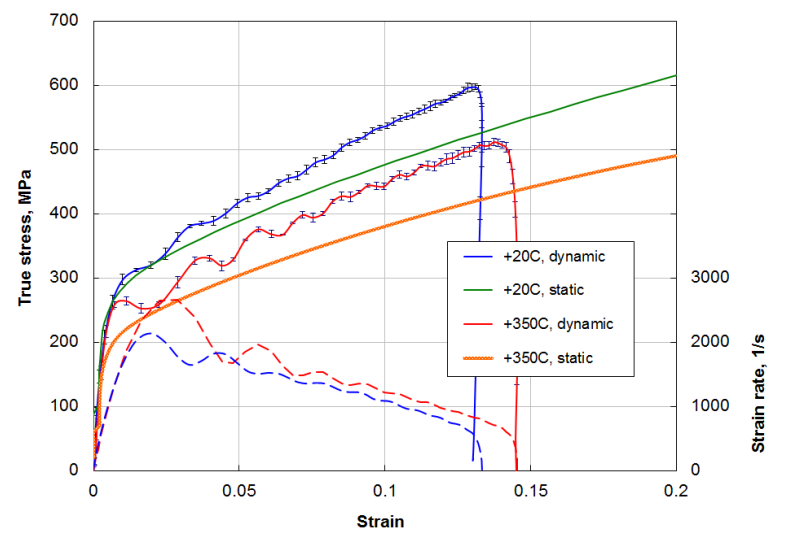

Fig. 2. Average stress-strain curves for steel under compression at different temperatures.

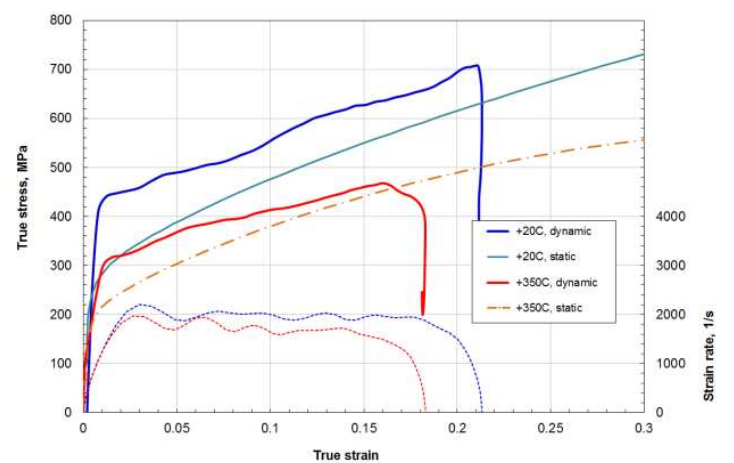

Fig. 3. Average stress-strain curves for steel under tension at different temperatures.

It can be seen that the temperature of the test has a significant effect on the mechanical properties: as the temperature rises, the diagrams go lower, and the steepness of the hardening section decreases. The 1810 steel showed a small (about $8 \%$ ) positive sensitivity of the yield point to an increase in the rate of deformation from static to dynamic values, and a negative (about $20 \%$ ) sensitivity to a rise in temperature from room temperature up to $+350{ }^{\circ} \mathrm{C}$.

To determine the limiting characteristics of ductility (relative elongation $\delta$ and relative narrowing $\psi$ after rupture), the folded halves of the disrupted sample were measured with a microscope and a digital eyepiece camera. The processing of the obtained images made it possible to measure the diameter of the neck and the residual length of the specimen. Based on the measurement data, the limiting plasticity characteristics were calculated: $\delta=57 \%, \psi=71 \%$. It should be noted that the ultimate fracture characteristics of $\delta$ and $\psi$ are practically independent of the strain rate and temperature.

\section{Material model identification and verification}

Based on the results of a series of basic experiments, the parameters of the Johnson-Cook plasticity model [7] were identified, which is a purely empirical determining equation for metals subject to large deformations, high strain rates and high temperatures. In this model, the flow stress in the multiplicative representation of the Mises yield surface is written as follows:

$$
\sigma=\left(A+B \varepsilon_{p}^{n}\right)\left(1+C \ln \varepsilon_{p}^{*}\right)\left(1-T^{* m}\right), \quad T^{*}=\frac{T-T_{\mathrm{a}}}{T_{m a l r}-T_{0}} .
$$

The five material constants $A, B, n, C$ and $m$ are determined empirically. The expression in the first brackets determines the dependence of the yield stress on the deformation at $\boldsymbol{z}^{*}=1$ and $T^{*}=0$. Expressions in the second and third brackets describe the effects of strain rate and temperature. In this model it is assumed that the effects of the influence of strain hardening, strain rate and temperature on the flow stress are mutually independent and, consequently, the stress does not depend on the loading path.

The problem of finding the optimal set of constants is a rather complex mathematical problem, since the minimized (efficiency) function is non-linear for four or more variables. For this function it is difficult to prove the existence of a single global minimum. However, since the function equipped with parameters and constants has a quite definite physical meaning, the minimization problem can be divided into a number of subtasks of smaller dimension.

At first, the plastic and strain hardening constants $(A$, $B, n)$ are determined from the isothermal tension (compression) test for relatively low strain rate $\left(\varepsilon^{*} \leq 1 \frac{1}{g}\right)$. The sensitivity $C$ to the strain rate is then determined from quasi-static and high-speed tests at different strain rates. Finally, the temperature softening constant $m$ is determined. 
Table 1. The parameters of models for the 1810 steel.

\begin{tabular}{|c|c|c|c|c|c|}
\hline Parameter & Model 1 & Model 2 & Model 3 & Model 4 & $\begin{array}{c}\text { Unit of } \\
\text { measure }\end{array}$ \\
\hline$A$ & 248.8 & 244 & 249 & 120 & MPa \\
\hline$B$ & 1339 & 1338 & 1339 & 648 & MPa \\
\hline$n$ & 0.6939 & 0.713 & 0.695 & 0.6958 & - \\
\hline$C$ & $8.18 \mathrm{E}-03$ & $7.49 \mathrm{E}-03$ & $8.18 \mathrm{E}-03$ & $1.53 \mathrm{E}-02$ & - \\
\hline$C_{2}$ & - & $8.22 \mathrm{E}-04$ & - & - & - \\
\hline$p$ & - & - & - & 63.288 & - \\
\hline$m$ & 1.18 & 1.164 & 1.179 & 1.178 & - \\
\hline
\end{tabular}

In [8] there was a shortage of the Johnson-Cook model related to the description of the dependence of the hardening work on the strain rate. In the latest releases of the LS-DYNA it became possible to use alternative forms of recording a high-speed multiplier (second brackets). In addition to the classical multiplier $1+C \cdot \ln \varepsilon_{y}^{*}$, logarithmic on strain rate, the quadratic factor proposed by Huh and Kang [9] is realized: $1+C \cdot \ln \left(\varepsilon^{4}\right)+C_{2} \cdot \ln \left(\varepsilon^{4}\right)^{2}$. Allen, Rule and Jones [10] suggested taking into account the velocity dependence of the yield strength using the following factor: $\left(\boldsymbol{z}^{*}\right)^{\mathrm{C}}$. It is also possible to describe the velocity dependence using the model proposed by Cowper and Symonds [11]: $1+\left(\mathrm{e}^{*} / C\right)^{1 / \mathrm{p}}$.

To identify the Johnson-Cook model with the four specified speed multipliers (hereinafter referred to as model 1-model 4, respectively), the results of a series of static and dynamic experiments for compression and tension at various temperatures were used. The parameters obtained are presented in Table 1.

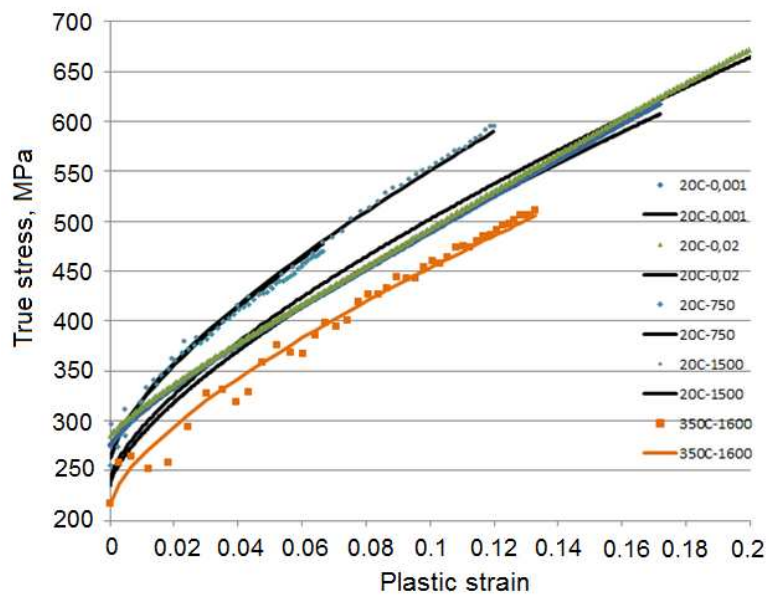

Fig. 4. Modeling of the 1810 steel behavior.

It is determined that the model 4 for this material gives the best approximation to the experimental data: the minimum deviation of the mathematical curve from the experimental stress-strain chart. Fig. 4 shows the comparison of deformation curves calculated in accordance with model 4 (solid lines), with experimental deformation charts (markers) obtained under different loading conditions (strain rate and temperature).
The obtained model of elastic-plastic behavior of the 1810 steel under high-speed loading was validated. To this end, a series of original verification experiments was carried out in the SHPB system in a natural and corresponding numerical implementation [12] including the dynamic indentation of indenters with a conical (angle at the apex of $90^{\circ}$ ) and hemispherical head, as well as diametrical elastic-plastic compression of the specimen (analog of the Brazilian test).

In the latter case, the modeling problem was not axisymmetric and must have been solved in a threedimensional formulation. When simulating experiments on high-speed indentation, contact between the indenter and the sample was established with friction (the coefficient of friction is 0.1 ).

a)
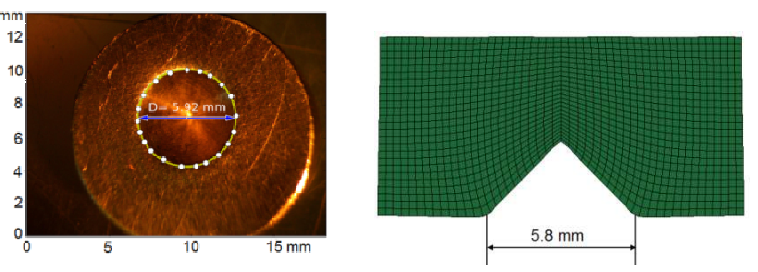

b)
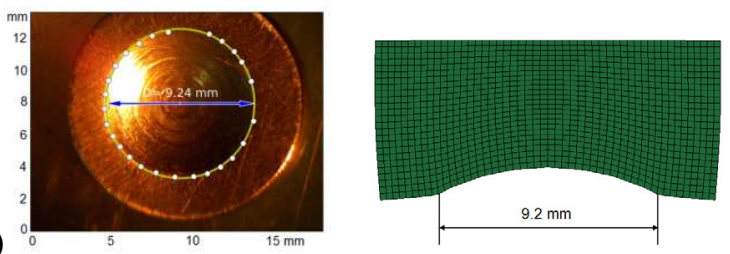

Fig. 5. Comparison of the diameter of the imprint obtained in the indentation natural experiment and after numerical modeling: $a$ - a conical indenter, $b$ - a hemispherical indenter.
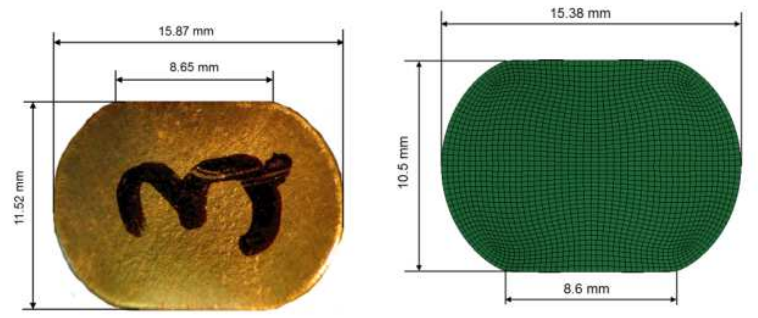

Fig. 6. Comparison of the residual shape of the sample obtained in the experiment on the diametrical compression and as a result of numerical simulation.

The degree of reliability of the model was determined by comparing the residual shape of the 
samples after loading in the full-scale (natural) test and in an appropriate numerical experiment. The results of comparison of the dent diameters in the specimens during the tests for the dynamic indentation of the conic and hemispherical indenters, as well as the residual shape of the specimens during the tests on diametrical compression are shown in Figs 5 and 6.

In addition, the strain pulses from the loading and supporting measuring bars, recorded in the experiment and obtained in numerical calculation, were compared.

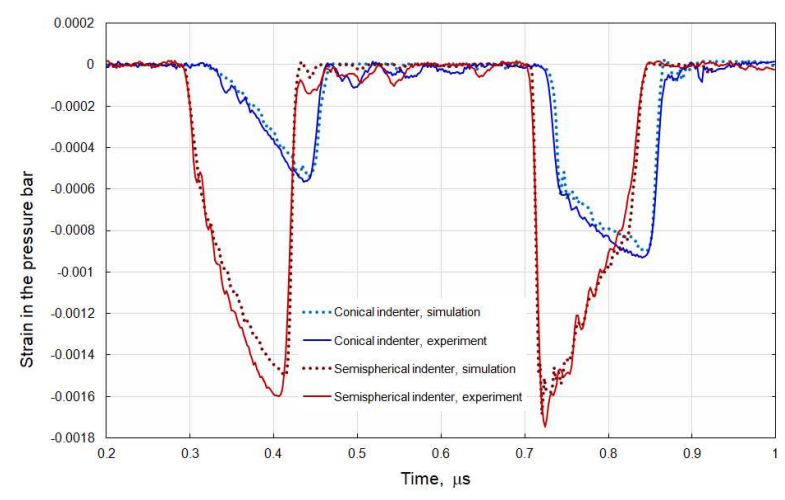

Fig. 7. Comparison of experimental data and calculation.

Figure 7 compares the pulses in the transmitting bar obtained in the natural test (solid lines) and in a numerical experiment (dashed lines) in the study of the indentation of conical and hemispherical indenters. We can see a fairly good correspondence of the signals, which indicates the reliability of the material model. The difference between the experimental and calculated data is less than $5 \%$.

Due to the small contact area of the indenter (especially the conical) with the specimen at the initial moment of the test, most of the loading wave was reflected back and after a while the specimen was loaded again. The test setup, due to the different lengths of the measuring bars [13], allowed reliably record two load cycles. For the conic indenter, the load amplitude in the second cycle is significant, and the implementation process is considerable. When using a hemispherical indenter, the contact area is much larger, so the main plastic deformation of the specimen occurs during the first load cycle.

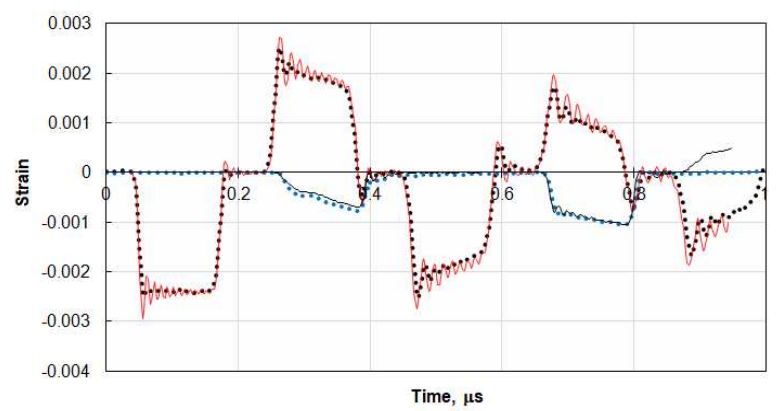

Fig. 8. Comparison of experimental data and calculation for diametrical compression.

Fig. 8 compares the deformation pulses from the incident and transmitting pressure bars registered in the experiment (markers) and the ones obtained in the calculation (solid line). Black markers and a red line represent information from the loading (incident) bar, blue markers and a black line - signals from the support (transmitting) bar.

As a result of validation it is determined that all four models allow reproducing quite accurately the results of the verification test. The results of a series of verification experiments for various types of stress-strain state indicate a high degree of reliability of all options for the model of behavior of stainless steel, but the best match is observed for model 4 with the speed multiplier proposed by Cowper and Symonds. The results of a series of verification experiments for various types of stress-strain state for this model show a good correspondence of the physical test data and numerical simulation with respect to the residual forms of the specimens and the time characteristics of the dynamics of the plastic deformation process, which are represented by data from the measuring bars, which indicates a high degree of reliability of the identified model.

In addition to the validation function of the mathematical model, full-scale experiments on the indentation make it possible to determine such an important mechanical characteristic of the material as dynamic hardness $[14,15]$. The procedure for hardness determining is as follows. The registration of the incident $\varepsilon^{i}(t)$ and reflected $\varepsilon^{r}(t)$ pulses makes it possible, on the basis of the one-dimensional theory of propagation of elastic waves, to determine the depth of indentation of the indenter into the specimen:

$$
h(t)=c \int_{o}^{t}\left[\varepsilon^{i}(t)-\varepsilon^{r}(t)\right] \cdot d t
$$

This ratio, in the case of the known geometry of the indenter, makes it possible to calculate the imprint surface area at any time during the application of the load. For a conic indenter with an angle at the vertex $2 \alpha$, the area $S(t)$ of the unrestored imprint is expressed as follows: $S(t)=\pi h^{2}(t) \frac{\operatorname{tg}^{2} \alpha}{\sin \alpha}$. In the case of using an indenter with a hemispherical head of diameter $\mathrm{D}$, the dent area is calculated by the formula: $S(t)=\pi D h(t)$.

The pulse $\varepsilon^{t}(t)$ in the transmitting bar makes it possible to determine the process of development of the force $F(t)$ in time, which occurs when the indenter is impressing into the specimen: $F(t)=E A \varepsilon^{t}(t)$, where $E$ is Young's modulus and $A$ is the area of the transverse section of the transmitting bar.

On the basis of the above dependences, it is possible to determine the dynamic Brinnel hardness $H B(t)$, as the ratio of the acting resistance force to the indenter onto the dent surface area: $H B(t)=\frac{F(t)}{S(t)}$. Thus, it is possible to calculate the value of $H B$ at any time in the indentation process.

For reliable calculation of dynamic hardness, the initial parametric dependences of $F(t)$ and $S(t)$, i.e. the pulses $\varepsilon^{i}(t), \varepsilon^{r}(t)$ and $\varepsilon^{t}(t)$ must be strictly consistent in time. In order to facilitate this synchronization, strain 
gauges were glued to measuring bars at the same distance from the specimen.

Figure 9 shows the dependencies of the resistance force at indentation and hardness of a material on the area of an imprint during two loading cycles using conic (a) and hemispherical $(b)$ indenters with an impression speed of $\sim 20 \mathrm{~m} / \mathrm{s}$, using the implemented technique. The tangent of the slope of the curve $F \sim S$ is an indicator of the dynamic hardness.

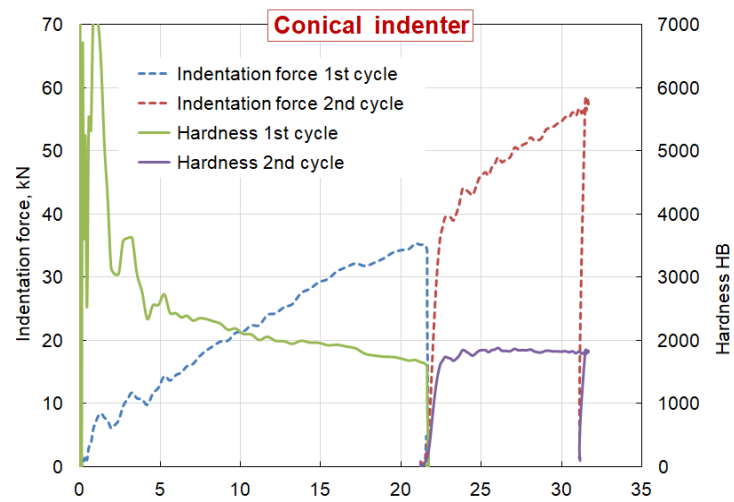

a)

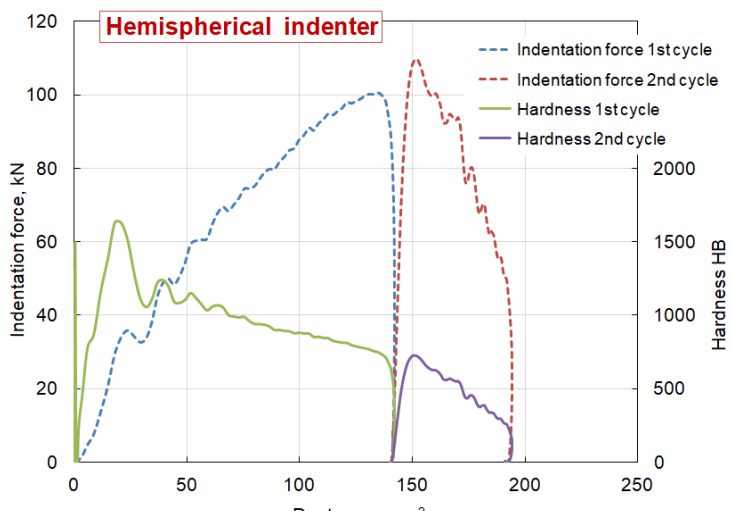

b)

Fig. 9. The results of investigation of dynamic hardness in the experiments on the indentation of the conical (a) and hemispherical (b) indenter.

The advantage of the proposed method is that the process of the dynamic hardness changing is registered during the entire indentation process.

\section{Summary}

The design of a simple gas gun was developed to create a direct tensile load in the SHPB using a tubular striker. A complex of dynamic tests of 1810 steel under compression and tension at normal and elevated temperatures was carried out.
Using a series of verification experiments for various types of stress-strain state, the degree of reliability of the identified mathematical model of the behavior of the material studied was determined. It is shown that the Johnson-Cook model, determined with a simple onedimensional stress state, reliably describes the elasticplastic behavior of steel under a complex stress state.

Experimental studies were carried out with the financial support of the grant of the Government of the Russian Federation (contract No.14.Y26.31.0031). Verification of mathematical model was carried out with partial financial support of the RSCF (grant No.16-19-10237).

\section{References}

1. B. Jiang, R. Zhang, J. Phys. IV, 134, 1071 (2006)

2. H. Eskandari, J.A. Nemes, J. Comp. Mater., 34, 4 (2000)

3. E. Cadoni, A. Bragov, M. Dotta, D. Forni, A. Konstantinov, A. Lomunov, A. Ripamonti, Eng. Trans. 59, 101 (2011)

4. J. Harding, in: M.A. Meyers et al. (Eds.), ShockWave and High Strain Rate Phenomena in Materials (Dekker, New York, 1992)

5. U.S. Lindholm, L.M. Yeakley, Exp. Mech. 8, 1 (1968)

6. T. Nicholas, Exp.Mech. 21, 177 (1981)

7. G.R. Johnson, W.H. Cook, Seventh International Symposium on Ballistic, 541 (1983)

8. S. Frechard, A. Lichtenberger, F. Rondot, N. Faderl, A. Redjaimia, M. Adoum, J. Phys. IV, 110, 9 (2003)

9. H. Huh, W.J. Kang, Int. J. Vehicle Design, 30, 1 (2002)

10. D.J. Allen, W.K. Rule, S.E. Jones, Exp. Mech. 37, 333 (1997)

11. G.R. Cowper, P.S. Symonds, Applied Mathematics Report (Brown University, 1958)

12. A.V. Abramov, A.M. Bragov, A.K. Lomunov, A.Yu. Konstantinov, L. Kruszka, I.V. Sergeichev, J.Phys. IV. 134487 (2006)

13. A.M. Bragov, A.K. Lomunov, I.V. Sergeichev, J. Appl. Mech. Tech. Phys., 42, 1090 (2001)

14. A.M. Bragov, A.K. Lomunov, A.I. Sadyrin, Method for determination of material hardness. Patent RF No.1486878 (1989)

15. A.M. Bragov, A.K. Lomunov, Int. J. Imp. Engng. 16, $321(1995)$ 
